\title{
Analysis of the diet in a Spanish prison and the level of perception in a sample of prisoners
}

\author{
Varoucha-Azcarate AC \\ Primary Prison Healthcare Team (EAPP). La Roca del Vallés. Centro Penitenciario Quatre Camins. \\ La Roca del Vallés. Barcelona
}

\begin{abstract}
Introducción: An analysis is carried out of the diet provided at the Quatre Camins prison (Barcelona), where meals were modified in the first quarter of 2017 to provide a healthier diet.

Objectives: The new meals are described in relation to the most prevalent chronic and metabolic pathologies in the prison population and it is checked if they correspond to a healthy diet. The food offered at the prison shops and the perception of prisoners regarding prison food are also analysed.

Materials and methods: A mixed methodology study was carried out: a) a descriptive analysis of the new food offered by the kitchen service; and b) an analysis of the meals using the Spanish Healthy Eating Index (IASE) as a reference; as well as a description of the most prevalent pathologies in the prison. To determine the prisoners' perceptions, an ad hoc self-completed questionnaire was designed that consisted of four closed Likert questions and four open questions about the perception of prison food. The prisoners who responded to the questionnaire did so voluntarily, anonymously and under informed consent. Results: A sample of 22 prisoners was used. The score according to the IASE is 60.5 points. The assessments of the sample of 22 prisoners regarding quality, quantity and perception of diet as healthy does not reach pass.

Discussion: Access to safe water and food meets the requirements for healthiness, quality and quantity regulated by the current Prison Regulation. The results obtained suggest that the diet in the prison "needs changes" according to the IASE.
\end{abstract}

Key words: healthy diet, prisons, chronic disease, feeding behavior, public health, metabolic disease.

Text received: $27 / 03 / 2018$

\section{INTRODUCTION}

The diseases with the greatest incidence amongst the prison population in the last century were those related to the respiratory tract and malnutrition, and unhealthy conditions were a contributory or aggravating factor in such pathologies ${ }^{1}$. The Spanish Prison Regulations (Reglamento Penitenciario) ${ }^{2}$ in fact gave great importance to the inmates' diet. In article 226 of the Regulations it states that "the inmates in all prisons shall be provided with a suitably prepared diet, which should respond to the dietary requirements of the prison population and the specific needs of age, health, work, climate, costumes and, whenever possible, personal and religious convictions". It also establishes that:
Text accepted: 13/07/2018

- "the diet of ill inmates shall be subject to medical control".

- "in those prisons where children accompany their mothers, the necessary resources shall be provided to feed each child in accordance with their needs and as per the instructions of the medical service" 2

The situation in Catalonia regarding concern for nutrition of the prison population is very similar. The Autonomous Community has had powers since 1983 over the management of prisons after transfer under Royal Decree 3482/83 of 28 December, and the concern over diet can be seen in article 89 of Decree 329/2006, of 5 September ${ }^{3}$, which specifies:

- That "healthcare services of the establishment shall control and ensure that the inmates' diet is 
a balanced one that responds to adequate criteria of nutrition, and that conforms in all cases with the dietary requirements of the prison population and specific health needs and that respects personal and religious convictions".

- That "the diet of ill inmates shall be subject to specific supervisions by the medical services of the prison, as well as that of minors who, in accordance with prison legislation, live with their mothers".

There are few previous studies about the analysis of diet in Spanish prisons. A study ${ }^{4}$ carried out at the old Barcelona Modelo prison showed after a detailed analysis of quality, quantity, temperature, variety, taste, noise and lighting, that the worst opinion about the food dispensed was reserved for the taste, followed by the variety. What was even more important was that the analysis of the diet, according to the Spanish Healthy Diet Index (IASE) , gave a rating of "acceptable" without being "healthy".

In Catalonia the collective prison kitchens are managed by a public company of the Regional Government of Catalonia: the Centre of Initiatives for

Table 1. Conversion of the new range of CIRE meals, from February 2017 onwards.

\begin{tabular}{|c|c|c|}
\hline Previous CIRE diet & Current CIRE diet & Supplements \\
\hline Astringent & Astringent & \\
\hline Astringent without fish & Astringent & \\
\hline Coeliac & Specific medical diet: coeliac & \\
\hline Soft without fish & Specific medical diet: soft W/F & \\
\hline General & General & \\
\hline $\begin{array}{l}\text { Diabetic supplement: } 1 \text { piece of fruit }+4 \text { crackers } \\
\text { (ham /light cheese) }\end{array}$ & Special & Only diabetics 2 times/day \\
\hline Special/Moslem & Special & \\
\hline Diabetic & Special & Diabetic Supplement \\
\hline Diabetic/Moslem & Special & Diabetic Supplement \\
\hline Liver/high blood pressure & Special & \\
\hline Liver/Moslem & Special & \\
\hline Special without fish & Special without fish & \\
\hline Diabetic/without fish & Special without fish & \\
\hline Liver/Moslem/without fish & Special without fish & \\
\hline Liver/without fish & Special without fish & \\
\hline Moslem/without fish & Without fish & \\
\hline General/without fish & Without fish & \\
\hline Moslem & Moslem & \\
\hline Moslem Ramadan & Moslem & \\
\hline Diabetic soft & Soft & Diabetic Supplement \\
\hline Soft/minced & Soft & Minced \\
\hline Soft/Moslem & Soft & \\
\hline Vegetarian & Vegetarian & \\
\hline Soft bread & & Soft bread \\
\hline One glass of fruit juice/not milk & Dairy free & \\
\hline One glass of sugar-free fruit juice & Dairy free & \\
\hline 1 skimmed milk yoghurt lunch & & Skimmed milk yoghurt lunch \\
\hline 2 yoghurt, instead of fruit lunch & & 2 yoghurt \\
\hline Allergic to lactose & $\begin{array}{l}\text { Specific medical diet: allergic to } \\
\text { lactose }\end{array}$ & \\
\hline
\end{tabular}

Note. CIRE: Centre d’Iniciatives per a la Reinserció. 
Varoucha-Azcarate AC. Analysis of the diet in a Spanish prison and the level of perception in a sample of prisoners.

Table 2. CIRE weekly meal planning

\begin{tabular}{|c|c|c|c|c|c|c|c|}
\hline Diet & Monday & Tuesday & Wednesday & Thursday & Friday & Saturday & Sunday \\
\hline \multicolumn{8}{|l|}{ Breakfast } \\
\hline General & $\begin{array}{c}\text { White coffee, } \\
\text { turkey slices, } \\
\text { pastries }\end{array}$ & $\begin{array}{c}\text { White coffee, } \\
\text { turkey slices, } \\
\text { pastries }\end{array}$ & $\begin{array}{c}\text { White coffee, } \\
\text { turkey slices, } \\
\text { pastries }\end{array}$ & $\begin{array}{c}\text { White coffee, } \\
\text { turkey slices, } \\
\text { pastries }\end{array}$ & $\begin{array}{c}\text { White coffee, } \\
\text { turkey slices, } \\
\text { pastries }\end{array}$ & $\begin{array}{c}\text { White coffee, } \\
\text { turkey slices, } \\
\text { pastries }\end{array}$ & $\begin{array}{c}\text { White coffee, } \\
\text { turkey slices, } \\
\text { pastries }\end{array}$ \\
\hline Moslem & $\begin{array}{c}\text { White coffee, } \\
\text { turkey slices, } \\
\text { pastries }\end{array}$ & $\begin{array}{c}\text { White coffee, } \\
\text { turkey slices, } \\
\text { pastries }\end{array}$ & $\begin{array}{c}\text { White coffee, } \\
\text { turkey slices, } \\
\text { pastries }\end{array}$ & $\begin{array}{c}\text { White coffee, } \\
\text { turkey slices, } \\
\text { pastries }\end{array}$ & $\begin{array}{c}\text { White coffee, } \\
\text { turkey slices, } \\
\text { pastries }\end{array}$ & $\begin{array}{c}\text { White coffee, } \\
\text { turkey slices, } \\
\text { pastries }\end{array}$ & $\begin{array}{c}\text { White coffee, } \\
\text { turkey slices, } \\
\text { pastries }\end{array}$ \\
\hline Vegetarian & $\begin{array}{l}\text { White coffee, } \\
\text { Edam cheese }\end{array}$ & $\begin{array}{l}\text { White coffee, } \\
\text { Edam cheese }\end{array}$ & $\begin{array}{l}\text { White coffee, } \\
\text { Edam cheese }\end{array}$ & $\begin{array}{l}\text { White coffee, } \\
\text { Edam cheese }\end{array}$ & $\begin{array}{l}\text { White coffee, } \\
\text { Edam cheese }\end{array}$ & $\begin{array}{l}\text { White coffee, } \\
\text { Edam cheese }\end{array}$ & $\begin{array}{l}\text { White coffee, } \\
\text { Edam cheese }\end{array}$ \\
\hline Special & $\begin{array}{c}\text { White coffee, } \\
\text { turkey slices, } \\
\text { pastries }\end{array}$ & $\begin{array}{c}\text { White coffee, } \\
\text { turkey slices, } \\
\text { pastries }\end{array}$ & $\begin{array}{c}\text { White coffee, } \\
\text { turkey slices, } \\
\text { pastries }\end{array}$ & $\begin{array}{c}\text { White coffee, } \\
\text { turkey slices, } \\
\text { pastries }\end{array}$ & $\begin{array}{c}\text { White coffee, } \\
\text { turkey slices, } \\
\text { pastries }\end{array}$ & $\begin{array}{c}\text { White coffee, } \\
\text { turkey slices, } \\
\text { pastries }\end{array}$ & $\begin{array}{c}\text { White coffee, } \\
\text { turkey slices, } \\
\text { pastries }\end{array}$ \\
\hline Soft & $\begin{array}{c}\text { White coffee, } \\
\text { turkey slices, } \\
\text { pastries }\end{array}$ & $\begin{array}{c}\text { White coffee, } \\
\text { turkey slices, } \\
\text { pastries }\end{array}$ & $\begin{array}{c}\text { White coffee, } \\
\text { turkey slices, } \\
\text { pastries }\end{array}$ & $\begin{array}{c}\text { White coffee, } \\
\text { turkey slices, } \\
\text { pastries }\end{array}$ & $\begin{array}{c}\text { White coffee, } \\
\text { turkey slices, } \\
\text { pastries }\end{array}$ & $\begin{array}{c}\text { White coffee, } \\
\text { turkey slices, } \\
\text { pastries }\end{array}$ & $\begin{array}{c}\text { White coffee, } \\
\text { turkey slices, } \\
\text { pastries }\end{array}$ \\
\hline Without fish & $\begin{array}{c}\text { White coffee, } \\
\text { turkey slices, } \\
\text { pastries }\end{array}$ & $\begin{array}{c}\text { White coffee, } \\
\text { turkey slices, } \\
\text { pastries }\end{array}$ & $\begin{array}{c}\text { White coffee, } \\
\text { turkey slices, } \\
\text { pastries }\end{array}$ & $\begin{array}{c}\text { White coffee, } \\
\text { turkey slices, } \\
\text { pastries }\end{array}$ & $\begin{array}{c}\text { White coffee, } \\
\text { turkey slices, } \\
\text { pastries }\end{array}$ & $\begin{array}{c}\text { White coffee, } \\
\text { turkey slices, } \\
\text { pastries }\end{array}$ & $\begin{array}{c}\text { White coffee, } \\
\text { turkey slices, } \\
\text { pastries }\end{array}$ \\
\hline \multirow[t]{2}{*}{ Dairy free } & $\begin{array}{c}\text { Fruit juice } \\
\text { with no added } \\
\text { sugar }\end{array}$ & $\begin{array}{c}\text { Fruit juice } \\
\text { with no added } \\
\text { sugar }\end{array}$ & $\begin{array}{l}\text { Fruit juice with } \\
\text { no added sugar }\end{array}$ & $\begin{array}{c}\text { Fruit juice } \\
\text { with no added } \\
\text { sugar }\end{array}$ & $\begin{array}{c}\text { Fruit juice } \\
\text { with no added } \\
\text { sugar }\end{array}$ & $\begin{array}{c}\text { Fruit juice } \\
\text { with no added } \\
\text { sugar }\end{array}$ & $\begin{array}{l}\text { Fruit juice with } \\
\text { no added sugar }\end{array}$ \\
\hline & $\begin{array}{c}\text { Turkey slices, } \\
\text { pastries }\end{array}$ & $\begin{array}{c}\text { Turkey slices, } \\
\text { pastries }\end{array}$ & $\begin{array}{c}\text { Turkey slices, } \\
\text { pastries }\end{array}$ & $\begin{array}{c}\text { Turkey slices, } \\
\text { pastries }\end{array}$ & $\begin{array}{c}\text { Turkey slices, } \\
\text { pastries }\end{array}$ & $\begin{array}{c}\text { Turkey slices, } \\
\text { pastries }\end{array}$ & $\begin{array}{c}\text { Turkey slices, } \\
\text { pastries }\end{array}$ \\
\hline \multirow[t]{2}{*}{ Astringent } & $\begin{array}{c}\text { Fruit juice } \\
\text { with no added } \\
\text { sugar }\end{array}$ & $\begin{array}{c}\text { Fruit juice } \\
\text { with no added } \\
\text { sugar }\end{array}$ & $\begin{array}{l}\text { Fruit juice with } \\
\text { no added sugar }\end{array}$ & $\begin{array}{c}\text { Fruit juice } \\
\text { with no added } \\
\text { sugar }\end{array}$ & $\begin{array}{c}\text { Fruit juice } \\
\text { with no added } \\
\text { sugar }\end{array}$ & $\begin{array}{c}\text { Fruit juice } \\
\text { with no added } \\
\text { sugar }\end{array}$ & $\begin{array}{l}\text { Fruit juice with } \\
\text { no added sugar }\end{array}$ \\
\hline & $\begin{array}{c}\text { Turkey slices, } \\
\text { pastries }\end{array}$ & $\begin{array}{c}\text { Turkey slices, } \\
\text { pastries }\end{array}$ & $\begin{array}{c}\text { Turkey slices, } \\
\text { pastries }\end{array}$ & $\begin{array}{c}\text { Turkey slices, } \\
\text { pastries }\end{array}$ & $\begin{array}{c}\text { Turkey slices, } \\
\text { pastries }\end{array}$ & $\begin{array}{c}\text { Turkey slices, } \\
\text { pastries }\end{array}$ & $\begin{array}{c}\text { Turkey slices, } \\
\text { pastries }\end{array}$ \\
\hline \multirow[t]{2}{*}{$\begin{array}{l}\text { Diabetic } \\
\text { supplement }\end{array}$} & $\begin{array}{c}\text { Crackers, } \\
\text { ham/light } \\
\text { cheese }\end{array}$ & $\begin{array}{c}\text { Crackers, } \\
\text { ham/light } \\
\text { cheese }\end{array}$ & $\begin{array}{l}\text { Crackers, ham/ } \\
\text { light cheese }\end{array}$ & $\begin{array}{c}\text { Crackers, } \\
\text { ham/light } \\
\text { cheese }\end{array}$ & $\begin{array}{c}\text { Crackers, } \\
\text { ham/light } \\
\text { cheese }\end{array}$ & $\begin{array}{c}\text { Crackers, } \\
\text { ham/light } \\
\text { cheese }\end{array}$ & $\begin{array}{l}\text { Crackers, ham/ } \\
\text { light cheese }\end{array}$ \\
\hline & 1 piece of fruit & 1 piece of fruit & 1 piece of fruit & 1 piece of fruit & 1 piece of fruit & 1 piece of fruit & 1 piece of fruit \\
\hline Lunch & & & & & & & \\
\hline General & $\begin{array}{l}\text { Rice casserole } \\
\text { with chicken }\end{array}$ & $\begin{array}{l}\text { Spaghetti a la } \\
\text { napolitana }\end{array}$ & $\begin{array}{c}\text { Lentils } \\
\text { with boiled } \\
\text { vegetables } \\
\end{array}$ & $\begin{array}{l}\text { Mixed salad } \\
\text { with turkey }\end{array}$ & $\begin{array}{l}\text { Haricot beans } \\
\text { with greens }\end{array}$ & $\begin{array}{c}\text { Tagliatelle a la } \\
\text { napolitana }\end{array}$ & $\begin{array}{c}\text { Fisherman style } \\
\text { potatoes }\end{array}$ \\
\hline Moslem & $\begin{array}{l}\text { Rice casserole } \\
\text { with chicken }\end{array}$ & $\begin{array}{l}\text { Spaghetti a la } \\
\text { napolitana }\end{array}$ & $\begin{array}{c}\text { Lentils } \\
\text { with boiled } \\
\text { vegetables }\end{array}$ & $\begin{array}{l}\text { Mixed salad } \\
\text { with turkey }\end{array}$ & $\begin{array}{l}\text { Haricot beans } \\
\text { with greens }\end{array}$ & $\begin{array}{c}\text { Tagliatelle a la } \\
\text { napolitana }\end{array}$ & $\begin{array}{c}\text { Fisherman style } \\
\text { potatoes }\end{array}$ \\
\hline Vegetarian & $\begin{array}{l}\text { Rice casserole } \\
\text { with vegetables }\end{array}$ & $\begin{array}{l}\text { Spaghetti with } \\
\text { boiled tomato }\end{array}$ & $\begin{array}{c}\text { Lentils } \\
\text { with boiled } \\
\text { vegetables }\end{array}$ & $\begin{array}{l}\text { Mixed salad } \\
\text { with cheese }\end{array}$ & $\begin{array}{l}\text { Haricot beans } \\
\text { with greens }\end{array}$ & $\begin{array}{c}\text { Tagliatelle a la } \\
\text { napolitana }\end{array}$ & $\begin{array}{l}\text { Stewed potatoes } \\
\text { with vegetables }\end{array}$ \\
\hline Special & $\begin{array}{l}\text { Rice casserole } \\
\text { with vegetables }\end{array}$ & $\begin{array}{l}\text { Spaghetti with } \\
\text { boiled tomato }\end{array}$ & $\begin{array}{c}\text { Lentils } \\
\text { with boiled } \\
\text { vegetables }\end{array}$ & $\begin{array}{l}\text { Mixed salad } \\
\text { with cheese }\end{array}$ & $\begin{array}{l}\text { Haricot beans } \\
\text { with greens }\end{array}$ & $\begin{array}{c}\text { Tagliatelle a la } \\
\text { napolitana }\end{array}$ & $\begin{array}{l}\text { Stewed potatoes } \\
\text { with vegetables }\end{array}$ \\
\hline Soft & $\begin{array}{l}\text { Rice casserole } \\
\text { with vegetables }\end{array}$ & $\begin{array}{l}\text { Boiled } \\
\text { spaghetti }\end{array}$ & $\begin{array}{c}\text { Lentils } \\
\text { with boiled } \\
\text { vegetables }\end{array}$ & $\begin{array}{c}\text { Carrots, } \\
\text { potatoes and } \\
\text { onion }\end{array}$ & $\begin{array}{l}\text { Potato and } \\
\text { carrot purée }\end{array}$ & $\begin{array}{c}\text { Boiled } \\
\text { tagliatelle with } \\
\text { oil } \\
\end{array}$ & $\begin{array}{l}\text { Stewed potatoes } \\
\text { with vegetables }\end{array}$ \\
\hline Without fish & $\begin{array}{l}\text { Rice casserole } \\
\text { with vegetables }\end{array}$ & $\begin{array}{l}\text { Spaghetti with } \\
\text { boiled tomato }\end{array}$ & $\begin{array}{c}\text { Lentils } \\
\text { with boiled } \\
\text { vegetables }\end{array}$ & $\begin{array}{l}\text { Mixed salad } \\
\text { with cheese }\end{array}$ & $\begin{array}{l}\text { Haricot beans } \\
\text { with greens }\end{array}$ & $\begin{array}{c}\text { Tagliatelle a la } \\
\text { napolitana }\end{array}$ & $\begin{array}{l}\text { Stewed potatoes } \\
\text { with vegetables }\end{array}$ \\
\hline Astringent & $\begin{array}{l}\text { Rice casserole } \\
\text { with chicken }\end{array}$ & $\begin{array}{c}\text { Boiled } \\
\text { spaghetti }\end{array}$ & $\begin{array}{l}\text { Potato and } \\
\text { carrot purée }\end{array}$ & $\begin{array}{c}\text { Carrots, } \\
\text { potatoes and } \\
\text { onion } \\
\end{array}$ & $\begin{array}{l}\text { Potato and } \\
\text { carrot purée }\end{array}$ & $\begin{array}{c}\text { Boiled } \\
\text { tagliatelle with } \\
\text { oil } \\
\end{array}$ & $\begin{array}{l}\text { Potatoes carrots } \\
\text { and onion }\end{array}$ \\
\hline
\end{tabular}


Table 2. CIRE weekly meal planning (continuation)

\begin{tabular}{|c|c|c|c|c|c|c|c|}
\hline Diet & Monday & Tuesday & Wednesday & Thursday & Friday & Saturday & Sunday \\
\hline \multicolumn{8}{|l|}{ Main course } \\
\hline General & $\begin{array}{l}\text { Veal meatballs } \\
\text { with potatoes }\end{array}$ & $\begin{array}{l}\text { Andalusian } \\
\text { style hake } \\
\text { fillet }\end{array}$ & $\begin{array}{l}\text { Pork cheeks: } \\
\text { general }\end{array}$ & $\begin{array}{c}\text { Hake fillet } \\
\text { with Russian } \\
\text { salad }\end{array}$ & $\begin{array}{c}\text { Chicken } \\
\text { leg with } \\
\text { mushrooms } \\
\text { and potatoes }\end{array}$ & $\begin{array}{c}\text { Roast chicken } \\
\text { leg }\end{array}$ & $\begin{array}{c}\text { Turkey sausages } \\
\text { with tomato } \\
\text { sauce }\end{array}$ \\
\hline Moslem & $\begin{array}{l}\text { Veal meatballs } \\
\text { with potatoes }\end{array}$ & $\begin{array}{l}\text { Andalusian } \\
\text { style hake } \\
\text { fillet }\end{array}$ & $\begin{array}{c}\text { Roast chicken } \\
\text { leg }\end{array}$ & $\begin{array}{c}\text { Hake fillet } \\
\text { with Russian } \\
\text { salad }\end{array}$ & $\begin{array}{c}\text { Chicken } \\
\text { leg with } \\
\text { mushrooms } \\
\text { and potatoes }\end{array}$ & $\begin{array}{c}\text { Roast chicken } \\
\text { leg }\end{array}$ & $\begin{array}{c}\text { Turkey sausages } \\
\text { with tomato } \\
\text { sauce }\end{array}$ \\
\hline Vegetarian & $\begin{array}{l}\text { Pinto beans } \\
\text { with greens }\end{array}$ & $\begin{array}{c}\text { Sautéed } \\
\text { vegetables } \\
\text { with potatoes }\end{array}$ & $\begin{array}{c}\text { Sautéed } \\
\text { vegetables }\end{array}$ & $\begin{array}{l}\text { Haricot beans } \\
\text { with greens }\end{array}$ & $\begin{array}{c}\text { Brussels } \\
\text { sprouts in } \\
\text { mustard sauce }\end{array}$ & $\begin{array}{l}\text { Pinto beans } \\
\text { with greens }\end{array}$ & $\begin{array}{l}\text { Vegetables } \\
\text { sticks }\end{array}$ \\
\hline Special & $\begin{array}{l}\text { Boiled veal } \\
\text { meatballs }\end{array}$ & $\begin{array}{l}\text { Grilled hake } \\
\text { fillet }\end{array}$ & $\begin{array}{c}\text { Roast chicken } \\
\text { leg }\end{array}$ & $\begin{array}{c}\text { Hake fillet } \\
\text { with Russian } \\
\text { salad }\end{array}$ & $\begin{array}{c}\text { Chicken } \\
\text { leg with } \\
\text { mushrooms } \\
\text { and potatoes }\end{array}$ & $\begin{array}{c}\text { Roast chicken } \\
\text { leg }\end{array}$ & Turkey sausages \\
\hline Soft & $\begin{array}{l}\text { Boiled veal } \\
\text { meatballs }\end{array}$ & $\begin{array}{l}\text { Grilled hake } \\
\text { fillet }\end{array}$ & $\begin{array}{c}\text { Roast chicken } \\
\text { leg }\end{array}$ & $\begin{array}{c}\text { Hake fillet } \\
\text { with Russian } \\
\text { salad }\end{array}$ & $\begin{array}{c}\text { Chicken } \\
\text { leg with } \\
\text { mushrooms } \\
\text { and potatoes }\end{array}$ & $\begin{array}{c}\text { Roast chicken } \\
\text { leg }\end{array}$ & Turkey sausages \\
\hline Without fish & $\begin{array}{l}\text { Veal meatballs } \\
\text { with potatoes }\end{array}$ & $\begin{array}{l}\text { Poultry } \\
\text { sausages }\end{array}$ & $\begin{array}{c}\text { Roast chicken } \\
\text { leg }\end{array}$ & $\begin{array}{l}\text { Boiled veal } \\
\text { meatballs }\end{array}$ & $\begin{array}{c}\text { Chicken } \\
\text { leg with } \\
\text { mushrooms } \\
\text { and potatoes }\end{array}$ & $\begin{array}{c}\text { Roast chicken } \\
\text { leg }\end{array}$ & Turkey sausages \\
\hline Astringent & $\begin{array}{l}\text { Veal meatballs } \\
\text { with potatoes }\end{array}$ & $\begin{array}{l}\text { Poultry } \\
\text { sausages }\end{array}$ & Carrot purée & $\begin{array}{c}\text { Potatoes } \\
\text { carrots and } \\
\text { onion }\end{array}$ & $\begin{array}{c}\text { Potatoes } \\
\text { carrots and } \\
\text { onion } \\
\end{array}$ & $\begin{array}{c}\text { Boiled } \\
\text { tagliatelle with } \\
\text { oil }\end{array}$ & Turkey sausages \\
\hline Garnish & Roast tomato & $\begin{array}{c}\text { Roast bell } \\
\text { pepper }\end{array}$ & $\begin{array}{c}\text { Sautéed } \\
\text { vegetables }\end{array}$ & Russian salad & $\begin{array}{c}\text { Roast } \\
\text { courgette }\end{array}$ & Roast tomato & $\begin{array}{c}\text { Roast bell } \\
\text { pepper }\end{array}$ \\
\hline \multicolumn{8}{|l|}{ Dessert } \\
\hline General & Apple & Apple & Apple & Apple & Apple & Apple & Apple \\
\hline Moslem & Apple & Apple & Apple & Apple & Apple & Apple & Apple \\
\hline Vegetarian & Apple & Apple & Apple & Apple & Apple & Apple & Apple \\
\hline Special & Apple & Apple & Apple & Apple & Apple & Apple & Apple \\
\hline Soft & Baked apple & Baked apple & Baked apple & Baked apple & Baked apple & Baked apple & Baked apple \\
\hline Without fish & Apple & Apple & Apple & Apple & Apple & Apple & Apple \\
\hline Astringent & Apple & Apple & Apple & Apple & Apple & Apple & Apple \\
\hline Dinner & 1 st course & & & & & & \\
\hline General & $\begin{array}{c}\text { Fish and rice } \\
\text { soup }\end{array}$ & $\begin{array}{l}\text { Green beans } \\
\text { and potatoes }\end{array}$ & $\begin{array}{l}\text { Vegetable and } \\
\text { potato stew }\end{array}$ & $\begin{array}{l}\text { Vegetable and } \\
\text { pasta soup }\end{array}$ & $\begin{array}{l}\text { Vegetable and } \\
\text { rice soup }\end{array}$ & $\begin{array}{c}\text { Vegetable and } \\
\text { potato stew }\end{array}$ & $\begin{array}{c}\text { Fish and rice } \\
\text { soup }\end{array}$ \\
\hline Moslem & $\begin{array}{l}\text { Fish and rice } \\
\text { soup }\end{array}$ & $\begin{array}{l}\text { Green beans } \\
\text { and potatoes }\end{array}$ & $\begin{array}{l}\text { Vegetable and } \\
\text { potato stew }\end{array}$ & $\begin{array}{l}\text { Vegetable and } \\
\text { pasta soup }\end{array}$ & $\begin{array}{l}\text { Vegetable and } \\
\text { rice soup }\end{array}$ & $\begin{array}{l}\text { Vegetable and } \\
\text { potato stew }\end{array}$ & $\begin{array}{c}\text { Fish and rice } \\
\text { soup }\end{array}$ \\
\hline Special & $\begin{array}{l}\text { Vegetable and } \\
\text { rice soup }\end{array}$ & $\begin{array}{l}\text { Green beans } \\
\text { and potatoes }\end{array}$ & $\begin{array}{c}\text { Vegetable and } \\
\text { potato stew }\end{array}$ & $\begin{array}{l}\text { Vegetable and } \\
\text { pasta soup }\end{array}$ & $\begin{array}{l}\text { Vegetable and } \\
\text { rice soup }\end{array}$ & $\begin{array}{l}\text { Vegetable and } \\
\text { potato stew }\end{array}$ & $\begin{array}{l}\text { Vegetable and } \\
\text { rice soup }\end{array}$ \\
\hline Astringent & $\begin{array}{l}\text { Vegetable and } \\
\text { rice soup }\end{array}$ & $\begin{array}{l}\text { Potato and } \\
\text { carrot purée }\end{array}$ & $\begin{array}{l}\text { Boiled potatoes } \\
\text { carrots and } \\
\text { onion }\end{array}$ & $\begin{array}{l}\text { Vegetable and } \\
\text { rice soup }\end{array}$ & $\begin{array}{l}\text { Vegetable and } \\
\text { rice soup }\end{array}$ & $\begin{array}{l}\text { Boiled cous } \\
\text { cous }\end{array}$ & $\begin{array}{l}\text { Vegetable and } \\
\text { rice soup }\end{array}$ \\
\hline Without fish & $\begin{array}{l}\text { Vegetable and } \\
\text { rice soup }\end{array}$ & $\begin{array}{l}\text { Green beans } \\
\text { and potatoes }\end{array}$ & $\begin{array}{l}\text { Vegetable and } \\
\text { potato stew }\end{array}$ & $\begin{array}{l}\text { Vegetable and } \\
\text { pasta soup }\end{array}$ & $\begin{array}{l}\text { Vegetable and } \\
\text { rice soup }\end{array}$ & $\begin{array}{c}\text { Vegetable and } \\
\text { potato stew }\end{array}$ & $\begin{array}{l}\text { Vegetable and } \\
\text { rice soup }\end{array}$ \\
\hline Soft & $\begin{array}{l}\text { Vegetable and } \\
\text { rice soup }\end{array}$ & $\begin{array}{l}\text { Green beans } \\
\text { and potatoes }\end{array}$ & $\begin{array}{c}\text { Vegetable and } \\
\text { potato stew }\end{array}$ & $\begin{array}{l}\text { Vegetable and } \\
\text { pasta soup }\end{array}$ & $\begin{array}{l}\text { Vegetable and } \\
\text { rice soup }\end{array}$ & $\begin{array}{c}\text { Vegetable and } \\
\text { potato stew }\end{array}$ & $\begin{array}{l}\text { Vegetable and } \\
\text { rice soup }\end{array}$ \\
\hline Vegetarian & $\begin{array}{l}\text { Vegetable and } \\
\text { rice soup }\end{array}$ & $\begin{array}{l}\text { Green beans } \\
\text { and potatoes }\end{array}$ & $\begin{array}{l}\text { Vegetable and } \\
\text { potato stew }\end{array}$ & $\begin{array}{l}\text { Vegetable and } \\
\text { pasta soup }\end{array}$ & $\begin{array}{l}\text { Vegetable and } \\
\text { rice soup }\end{array}$ & $\begin{array}{c}\text { Vegetable and } \\
\text { potato stew }\end{array}$ & $\begin{array}{l}\text { Vegetable and } \\
\text { rice soup }\end{array}$ \\
\hline
\end{tabular}


Varoucha-Azcarate AC. Analysis of the diet in a Spanish prison and the level of perception in a sample of prisoners.

Table 2. CIRE weekly meal planning (continuation)

\begin{tabular}{|c|c|c|c|c|c|c|c|}
\hline Diet & Monday & Tuesday & Wednesday & Thursday & Friday & Saturday & Sunday \\
\hline \multicolumn{8}{|l|}{ Main course } \\
\hline General & $\begin{array}{l}\text { Turkey roll in } \\
\text { vegetable and } \\
\text { tomato sauce } \\
\text { with potatoes }\end{array}$ & $\begin{array}{l}\text { Potato } \\
\text { and onion } \\
\text { omelette }\end{array}$ & 4 cheese pizza & Veal burgers & $\begin{array}{l}\text { Chicken } \\
\text { sausages in } \\
\text { tomato sauce }\end{array}$ & $\begin{array}{l}\text { Veal meatballs } \\
\text { in tomato } \\
\text { sauce }\end{array}$ & Boiled eggs \\
\hline Moslem & $\begin{array}{l}\text { Turkey roll in } \\
\text { vegetable and } \\
\text { tomato sauce } \\
\text { with potatoes }\end{array}$ & $\begin{array}{l}\text { Potato } \\
\text { and onion } \\
\text { omelette }\end{array}$ & 4 cheese pizza & Veal burgers & $\begin{array}{c}\text { Chicken } \\
\text { sausages in } \\
\text { tomato sauce }\end{array}$ & $\begin{array}{l}\text { Veal meatballs } \\
\text { in tomato } \\
\text { sauce }\end{array}$ & Boiled eggs \\
\hline Special & $\begin{array}{c}\text { Turkey roll } \\
\text { with vegetables }\end{array}$ & $\begin{array}{l}\text { French } \\
\text { omelette }\end{array}$ & $\begin{array}{c}\text { Grilled chicken } \\
\text { breast }\end{array}$ & Veal burgers & $\begin{array}{l}\text { Chicken } \\
\text { sausages }\end{array}$ & $\begin{array}{l}\text { Boiled veal } \\
\text { meatballs }\end{array}$ & Boiled eggs \\
\hline Astringent & $\begin{array}{c}\text { Turkey roll } \\
\text { with vegetables }\end{array}$ & $\begin{array}{c}\text { French } \\
\text { omelette }\end{array}$ & $\begin{array}{c}\text { Grilled chicken } \\
\text { breast }\end{array}$ & Veal burgers & $\begin{array}{l}\text { Chicken } \\
\text { sausages }\end{array}$ & $\begin{array}{l}\text { Poultry } \\
\text { sausages }\end{array}$ & Boiled eggs \\
\hline Without fish & $\begin{array}{c}\text { Turkey roll } \\
\text { with vegetables }\end{array}$ & $\begin{array}{c}\text { French } \\
\text { omelette }\end{array}$ & $\begin{array}{c}\text { Grilled chicken } \\
\text { breast }\end{array}$ & Veal burgers & $\begin{array}{l}\text { Chicken } \\
\text { sausages }\end{array}$ & $\begin{array}{c}\text { Boiled veal } \\
\text { meatballs }\end{array}$ & Boiled eggs \\
\hline Soft & $\begin{array}{c}\text { Turkey roll } \\
\text { with vegetables }\end{array}$ & $\begin{array}{c}\text { French } \\
\text { omelette }\end{array}$ & $\begin{array}{c}\text { Grilled chicken } \\
\text { breast }\end{array}$ & Veal burgers & $\begin{array}{l}\text { Chicken } \\
\text { sausages }\end{array}$ & $\begin{array}{l}\text { Poultry } \\
\text { sausages }\end{array}$ & Boiled eggs \\
\hline Vegetarian & Escalivada* & $\begin{array}{c}\text { Potato } \\
\text { and onion } \\
\text { omelette } \\
\end{array}$ & 4 cheese pizza & $\begin{array}{l}\text { Potato filled } \\
\text { with spinach } \\
\text { au gratin }\end{array}$ & $\begin{array}{l}\text { Courgette } \\
\text { omelette }\end{array}$ & $\begin{array}{l}\text { Couscous } \\
\text { curry and } \\
\text { vegetables }\end{array}$ & Boiled eggs \\
\hline \multicolumn{8}{|l|}{ Garnish } \\
\hline General & & Salad & $\begin{array}{l}\text { Roast natural } \\
\text { tomato }\end{array}$ & Baked potato & Baked potato & Boiled rice & Escalivada* \\
\hline Moslem & & Salad & $\begin{array}{c}\text { Roast natural } \\
\text { tomato }\end{array}$ & Baked potato & Baked potato & Boiled rice & Escalivada* \\
\hline Special & & Salad & $\begin{array}{l}\text { Roast natural } \\
\text { tomato }\end{array}$ & Baked potato & Baked potato & Boiled rice & Escalivada* \\
\hline Astringent & & & & Baked potato & Baked potato & Boiled rice & Escalivada* \\
\hline Without fish & & Salad & $\begin{array}{l}\text { Roast natural } \\
\text { tomato }\end{array}$ & Baked potato & Baked potato & $\begin{array}{c}\text { Roast } \\
\text { courgette }\end{array}$ & Escalivada* \\
\hline Soft & & $\begin{array}{c}\text { Roast } \\
\text { courgette }\end{array}$ & $\begin{array}{c}\text { Roast } \\
\text { aubergine }\end{array}$ & Baked potato & Baked potato & $\begin{array}{c}\text { Roast } \\
\text { courgette }\end{array}$ & Escalivada* \\
\hline Vegetarian & & Salad & $\begin{array}{l}\text { Roast natural } \\
\text { tomato }\end{array}$ & & & $\begin{array}{c}\text { Roast } \\
\text { courgette }\end{array}$ & Escalivada* \\
\hline \multicolumn{8}{|l|}{ Desert } \\
\hline General & Apple & Apple & $\begin{array}{c}\text { Flavoured } \\
\text { yoghurt }\end{array}$ & $\begin{array}{c}\text { Flavoured } \\
\text { yoghurt }\end{array}$ & $\begin{array}{c}\text { Flavoured } \\
\text { yoghurt }\end{array}$ & $\begin{array}{c}\text { Flavoured } \\
\text { yoghurt }\end{array}$ & $\begin{array}{c}\text { Flavoured } \\
\text { yoghurt }\end{array}$ \\
\hline Moslem & Apple & Apple & $\begin{array}{c}\text { Flavoured } \\
\text { yoghurt }\end{array}$ & $\begin{array}{c}\text { Flavoured } \\
\text { yoghurt }\end{array}$ & $\begin{array}{c}\text { Flavoured } \\
\text { yoghurt }\end{array}$ & $\begin{array}{c}\text { Flavoured } \\
\text { yoghurt }\end{array}$ & $\begin{array}{c}\text { Flavoured } \\
\text { yoghurt }\end{array}$ \\
\hline Special & Apple & Apple & $\begin{array}{c}\text { Skimmed milk } \\
\text { yoghurt }\end{array}$ & $\begin{array}{c}\text { Skimmed milk } \\
\text { yoghurt }\end{array}$ & Apple & $\begin{array}{c}\text { Skimmed milk } \\
\text { yoghurt }\end{array}$ & $\begin{array}{c}\text { Skimmed milk } \\
\text { yoghurt }\end{array}$ \\
\hline Astringent & Apple & Apple & Apple & Apple & Apple & Apple & Apple \\
\hline Without fish & Apple & Apple & $\begin{array}{c}\text { Skimmed milk } \\
\text { yoghurt }\end{array}$ & $\begin{array}{c}\text { Skimmed milk } \\
\text { yoghurt }\end{array}$ & Apple & $\begin{array}{c}\text { Skimmed milk } \\
\text { yoghurt }\end{array}$ & $\begin{array}{c}\text { Skimmed milk } \\
\text { yoghurt }\end{array}$ \\
\hline Soft & Apple & Apple & $\begin{array}{l}\text { Skimmed milk } \\
\text { yoghurt }\end{array}$ & $\begin{array}{l}\text { Skimmed milk } \\
\text { yoghurt }\end{array}$ & Apple & $\begin{array}{l}\text { Skimmed milk } \\
\text { yoghurt }\end{array}$ & $\begin{array}{c}\text { Skimmed milk } \\
\text { yoghurt }\end{array}$ \\
\hline Vegetarian & Apple & Apple & $\begin{array}{c}\text { Flavoured } \\
\text { yoghurt }\end{array}$ & $\begin{array}{c}\text { Flavoured } \\
\text { yoghurt }\end{array}$ & $\begin{array}{c}\text { Flavoured } \\
\text { yoghurt }\end{array}$ & $\begin{array}{c}\text { Flavoured } \\
\text { yoghurt }\end{array}$ & $\begin{array}{c}\text { Flavoured } \\
\text { yoghurt }\end{array}$ \\
\hline
\end{tabular}

Note: Week selected at random. CIRE: Centre d'Iniciatives per a la Reinserció.

*Catalonian style roast veg. 
Reinsertion (Centre d'Iniciatives per a la Reinserció (CIRE)). In February 2017, the nutrition department of this company started to redesign the menus and unified many of the diets that had coexisted up till then. This study describes and analyses these new menus using a random gathering of the daily spreadsheets, the new diets offered are checked to see if they are healthy for the prison population and perceptions about the diets taken from a sample of inmates are described. Finally, and analysis is run to see if the new menus match the dietary recommendations for the chronic and metabolic pathologies that are most prevalent amongst the inmates.

\section{MATERIALS AND METHODS}

A cross-sectional descriptive study was carried out of the new diets offered by the kitchen service, managed by the public company CIRE, regarding the number of medically prescribed diets and in particular, the description of the diet called the "special diet”. The study was carried out at the Quatre Camins Prison (Barcelona), where approximately 1,400 adult males are interned. Table 1 describes the changes that the supply company began to implement in the first quarter of 2017 to the collective nutrition of Catalonian prisons and in particular, to the Quatre Camins Prison. A template was prepared to give a schematic view of the different meals offered on a given day and the respective dishes (Table 2).

A cross-section dated 26/4/2017 was carried out on the Prison IT System of Catalonia (Sistema Informático Penitenciario de Cataluña (SIPC) $)^{6}$ to obtain the diets prescribed by doctors. The analysis by groups of the food in these menus (Table 2), according to the IASE, is described in Table 3. Scores were given of the groups of foods of the daily menus randomly selected from the Department of Nursing/Psychiatry via the IASE. The distribution sheets of meals per module were obtained from the prison kitchen service. The IASE consists of ten variables by groups of foods: cereals-derivatives, vegetables-root vegetables, fruit, milk and derivatives, meat, legumes, sausage-cold meats, sweets, soft drinkssugar and variety-diet. The categories of the IASE5 have a maximum score of 100 and are classified as: "healthy nutrition", at more than 80 points; "needs changes", at between 50 and 80 points; "not healthy", at 50 points. This scales was drawn up from the Consumption Frequency Questionnaire (Cuestionario de Frecuencia de Consumo (CFC)) and from the recommendations of

Table 3. Quality of the Catalonian prison diet based on the meals selected in the CIRE weekly menu planning and scoresas per the IASE

\begin{tabular}{|c|c|c|c|c|c|}
\hline Variables & Daily (10) & $\begin{array}{c}3 \text { or more a } \\
\text { week }(7.5) \\
\end{array}$ & $\begin{array}{c}1 \text { or } 2 \text { a week } \\
(5)\end{array}$ & $\begin{array}{c}\text { Less than } 1 \mathrm{a} \\
\text { week }(2.5)\end{array}$ & $\begin{array}{c}\text { Never or } \\
\text { hardly ever (0) }\end{array}$ \\
\hline \multicolumn{6}{|l|}{ Daily consumption } \\
\hline 1. Cereals and wheat & 10 & & & & \\
\hline 2. Vegetables and root vegetables & 10 & & & & \\
\hline 3. Fruit & 10 & & & & \\
\hline 4. Milk and dairy products & 10 & & & & \\
\hline \multicolumn{6}{|l|}{ Weekly consumption } \\
\hline 5. Meat & 10 & & & & \\
\hline 6. Legumes & & 7,5 & & & \\
\hline \multicolumn{6}{|l|}{ Occasional consumption } \\
\hline 7. Cured sausage and cold & Daily (0) & & & & \\
\hline 8. Sweets & Daily (0) & & & & \\
\hline 9. Soft drinks with sugar & Daily (0) & & & & \\
\hline 10. Variety & 2 & 1 & & & \\
\hline TOTAL: & 60,5 & & & & \\
\hline RESULT: Needs changes & & & & & \\
\hline
\end{tabular}

Note. CIRE: Centre d'Iniciatives per a la Reinserció. IASE: Spanish Index of Healthy Nutrition (Índice de Alimentación Saludable Español). 
the Dietary Guides of the Spanish Society for Community Nutrition in 2004 (Guias Alimentarias de la Sociedad Española de Nutrición Comunitaria en 2004). The IASE is based on the methodology used for the HEI, or Healthy Eating Index, and this tool was selected for its usefulness in estimating the quality of the diet in a population, in this case the prison population of Quatre Camins.

The data base of the Primary Healthcare IT System of the Catalonian Health Institute (l'Institut Catalá de la Salut) e-Cap: DbsForm ("Health problems") was used for the diagnostic classification of pathologies. Inmates' perceptions were assessed qualitatively by means of a brief interview and completing an ad hoc questionnaire, structured one closed question, four with a value from 1 to 10 points, and three open questions, which were answered by a sample of 22 randomly selected inmates during the nursing examination. Participation was voluntary, anonymous and given under informed consent.

\section{RESULTS}

The manager and technical director of the food service of the CIRE informed the healthcare services of the prison via a corporate electronic mail message of the changes to be made to the inmates' diets, describing in detail what could be prescribed for medical reasons. The diet now called "special" replaces the previous diets issued under medical prescription, depending on the different medical pathologies, such as "diabetic", "high blood pressure ", "dyslipidemia” or "obesity".

The Special diet consists of a balanced diet, low in salt (1,500-2,000 mg/day of sodium) and with a heart-healthy lipid profile. It is a diet indicated for any pathology that requires restrictions on salt, that is in a compensated stage, in other words, without severe oedemas or abdominal fluid retention (ascites). In the opinion of the CIRE it is recommended for inmates with high blood pressure, excess weight, obesity or dyslipidemia. The diet can also be used for diabetic inmates as it distributes as part of its composition throughout the day a carbohydrate content of $50-55 \%$ of the total energy value (TEV) and is low in fast absorption sugars. Therefore, and according to the nutrition service of the CIRE, this diet excludes pork and uses less fatty, leaner meats such as veal, chicken or turkey. Fried dishes, stews and casseroles are sys- tematically excluded, and so the cooking processes used are without salt, grilled, roast or steamed, boiled in stew form using a dietary approach. The bread and crackers that accompany the dishes are salt free.

Breakfast for inmates with diabetes mellitus type 1 or 2 consists of a sandwich with turkey sausage meat or fat and salt free cheese. They are also given a hot drink. An additional supplement to the breakfast is two crackers with diet jam, and a piece of fruit for elevenses. When lunch is served, they receive four crackers with light cheese slices for an afternoon snack, and with the dinner, they are given a piece of fruit or skimmed milk yoghurt as a night time dietary supplement.

Other medically prescribed diets are not described since they are not associated with metabolic diseases, such as the "soft diet", "diet without fish", "astringent diet", "diet specific celiac diet" and "dairy free diet".

\section{Quantitative results}

There were a total of 348 diets prescribed for a population of 1,428 inmates $(24.3 \%)$, of which 146 were "special” $(10.5 \%)^{6}$.

The score of the analysis by groups of foods was 60.5 points; or rather, a score defined as "needs changes", according to the categories of the IASE 5 .

As regards frequency of consumption, in the sweets category, the score assigned is 0 , bearing in mind the criteria for the minimum score of this variable and that the kitchen service prepares and serves pastries every day at breakfast.

In the category of sausages and cold meats, the one served every day is turkey luncheon meat, and so the score has also been classified in the column of "daily consumption" with a score of 0 ; if it was "never or hardly ever", the score would be 10 .

2 points were assigned to the criteria "variety", as it the recommended daily consumption of the groups of foods is complied with, although this criterion does not correlated with the perception of variety mentioned by the inmates in the interviews.

Table 4 shows the prevalence of chronic and metabolic diseases amongst the inmates of Quatre Camins prison. $43.6 \%$ of the inmates would be candidates for receiving a "special" diet, but only $10.5 \%$ of them had the diet prescribed.

\section{Qualitative results}

$40 \%$ of the diners did not notice any substantial changes to the menus in recent months, stating that 
"it's always bad". As regard the quality, quantity and if they considered it to be healthy, the average score did not reach pass (4.5 points), which underlines the negative perception of the prisoners in these three areas.

$92 \%$ of the interviewees answered that they bought food at the prison shop. $8 \%$ of the interviewees declared that they could not buy any food or drinks because they did not have the means to do so, and so only eat the food offered by the prison three times a day. It was asked why the prisoners consume products from the shop and the answers included: "to have some variety", "because of the lack of food", "because the food is awful", "to eat more", "to compensate", "because I don't get enough with breakfast, lunch and dinner", "because I feel like it", and "because I'm not satisfied with the food". One of the interviewees stated that "I don't eat anything the prison offers because I don't like what there is and because I can buy food" and said that many inmates do the same as he does.

\section{DISCUSSION}

Following the ethnographic lines of the article by Fornons ${ }^{7}$, after the changes established in the diet of the CIRE, it could be seen that pork and pork products were removed from all the diets, regardless of the beliefs professed by the inmate, to make it "healthier". Only roast pork cheeks are served twice a month as a special dish in the general diet. The fact that this type of meat has been removed from all the diets is a measure that solves the problems of cooking processes crossed with oils or surfaces in contact with different raw materials.

In the study by Sáiz-Izquierdo et $\mathrm{al}^{4}{ }^{4}$, negative assessments were given by inmates of the quality, taste, temperature and hardness of the meat, although they did not appear to object to the quantity, since they explained that they could have second helpings. Despite the changes made by the Dietetics and Nutrition Service of the CIRE in 2017, the diet offered only obtains 60.5 points and therefore the evaluation is one of "needs changes". The outcome obtained by Sáiz-Izquierdo et al. ${ }^{4}$ was 58.4 points, a very similar result, and the researchers suggested changes to improve the scores, which included reducing the consumption of meat and increasing legumes as a main course. This measure could be a good choice, as one of the main complaints from inmates is in fact the hardness or undercooking of the meat. The proposed measure could therefore be an effective one since legumes are tasty, healthy and economical, and the prison would benefit by having a cost-sustainable menu.

It is also interesting to comment on the culinary and cooking techniques, since it is suggested that fry-

Table 4. Most prevalent pathologies at the Quatre Camins Prison

\begin{tabular}{lccccc}
\hline Obesity BMI $>30$ & Dyslipedemia & Hepatitis C & $\begin{array}{c}\text { High blood } \\
\text { pressure }\end{array}$ & HIV & $\begin{array}{c}\text { Diabetes type 1 } \\
\text { and 2 }\end{array}$ \\
\hline $16.3 \%$ & $13.19 \%$ & $11.4 \%$ & $9.51 \%$ & $6.6 \%$ & $4.1 \%$ \\
\hline
\end{tabular}

Note. Total population of 1,378 inmates on the cut-off date of $27 / 3 / 2017$, en total $601,43.6 \%$ of the inmates with metabolic pathologies (in percentage terms). The most prevalent pathologies appear in dark grey below.

HIV: human immunodeficiency virus. BMI: body mass index.

Table 5. Description and analysis of the surveys on perception of diet at the Quatre Camins Prison in a sample of 22 inmates.

\begin{tabular}{|c|c|c|c|}
\hline Level of satisfaction & Average 3.25 & & \\
\hline Sufficient quantity & Average 4.9 & & \\
\hline Healthy & Average 4.5 & & \\
\hline Buys food at the prison shop & $95 \%$ & & \\
\hline Reason for buying & Bad & Repetitive & Hungry \\
\hline Food bought & $\begin{array}{c}\text { Cheese } \\
\text { cured meats } \\
\text { Nuts }\end{array}$ & $\begin{array}{c}\text { Cans } \\
\text { Milk } \\
\text { Potato crisps }\end{array}$ & $\begin{array}{l}\text { Pre-baked } \\
\text { biscuits } \\
\text { All: }\end{array}$ \\
\hline $\begin{array}{l}\text { From all the food available at the prison, which one } \\
\text { would you like that is not offered. }\end{array}$ & $\begin{array}{l}\text { Fried food Variety } \\
\text { of yoghurt }\end{array}$ & Fruit Milkshake & Fried eggs \\
\hline
\end{tabular}


Varoucha-Azcarate AC. Analysis of the diet in a Spanish prison and the level of perception in a sample of prisoners.

Table 6. Food at the prison shop CIRE Quatre Camins.

\begin{tabular}{|c|c|c|}
\hline \multicolumn{3}{|c|}{ Food and drink } \\
\hline Fruit juice $330 \mathrm{ml}$ & $0 \%$ alcohol beer can $33 \mathrm{cl}$ & Horchata $1 \mathrm{~L}$ \\
\hline Coca-Cola tin $33 \mathrm{cl}$ & Font Vella water $1.5 \mathrm{~L}$ & $\begin{array}{c}\text { Font Vella Levité } \\
\text { Lemon drink 1.25 L }\end{array}$ \\
\hline \multicolumn{3}{|l|}{ Fruit juice/milk "Mediterráneo" $330 \mathrm{ml}$} \\
\hline \multicolumn{3}{|c|}{ Tinned foods } \\
\hline Stuffed olives $300 \mathrm{~g}$ & Sweetcorn $150 \mathrm{~g}$ & Squid American sauce $111 \mathrm{~g}$ \\
\hline Mussels in spicy sauce $112 \mathrm{~g}$ & Pate Mina $80 \mathrm{~g}$ & Sweet red pepper $80 \mathrm{~g}$ \\
\hline Tuna in hot sauce $111 \mathrm{~g}$ & Hot and spicy skewered olives $300 \mathrm{~g}$ & Turkey luncheon meat $400 \mathrm{~g}$ \\
\hline Clams in water $112 \mathrm{~g}$ & Tripe a la "madrileña" $380 \mathrm{~g}$ & Asturian bean stew $440 \mathrm{~g}$ \\
\hline Sliced pineapple $225 \mathrm{~g}$ & Fruit cocktail $220 \mathrm{~g}$ & Anchovies in oil 46g \\
\hline Asparagus $230 \mathrm{~g}$ & White tuna in oil $110 \mathrm{~g}$ & Mackerel in oil $90 \mathrm{~g}$ \\
\hline Country Russian salad $200 \mathrm{~g}$ & & Sardines in oil $120 \mathrm{~g}$ \\
\hline \multicolumn{3}{|c|}{ Food dairy products } \\
\hline Condensed milk $170 \mathrm{~g}$ & $\begin{array}{c}\text { Actimel yoghurt with strawberry and } \\
\text { banana }\end{array}$ & $\begin{array}{l}\text { Chocolate, strawberry, vanilla } \\
\text { milkshake 1 L }\end{array}$ \\
\hline Rice pudding & Cheese spread $200 \mathrm{~g}$ & Whole milk $1 \mathrm{~L}$ \\
\hline Yogures fibra y cereales & Tabla 4 quesos $150 \mathrm{~g}$ & Skimmed milk 1 L \\
\hline \multicolumn{3}{|c|}{ Cured sausage and meat } \\
\hline Selection of Iberian sausage $100 \mathrm{~g}$ & Ham $100 \mathrm{~g}$ & Chorizo extra $100 \mathrm{~g}$ \\
\hline \multicolumn{3}{|l|}{ Sobrasada $250 \mathrm{~g}$} \\
\hline \multicolumn{3}{|c|}{ General food } \\
\hline Salted popcorn $18 \mathrm{~g}$ & Potato crisps $30 \mathrm{~g}$ & Mayonnaise $14 \mathrm{~g}$ \\
\hline Chocolate cream $500 \mathrm{~g}$ & Cupcakes $150 \mathrm{~g}$ & Barquichoco biscuits $150 \mathrm{~g}$ \\
\hline Whole wheat María biscuits $200 \mathrm{~g}$ & Intense flavour olive oil $1 \mathrm{~L}$ & Country style crackers \\
\hline Gluten free white bread $300 \mathrm{~g}$ & Gazpacho $390 \mathrm{~g}$ & Cream of courgette soup $500 \mathrm{~g}$ \\
\hline Dates $200 \mathrm{~g}$ & Chocolate filled biscuits $180 \mathrm{~g}$ & Cream of mushroom soup $500 \mathrm{~g}$ \\
\hline Cough sweets $150 \mathrm{~g}$ & Trail mix $120 \mathrm{~g}$ & \\
\hline \multicolumn{3}{|c|}{ Peeled sunflower seeds $150 \mathrm{~g}$} \\
\hline & Sweets such as fruit gums, etc & \\
\hline
\end{tabular}

Note. CIRE: Centre d'Iniciatives per a la Reinserció.

ing and fats should be avoided and this measure was adopted by almost exclusively using oven cooking, grills or boiling, which possibly makes for a considerable improvement in terms of cardiac health, but which is hardly accepted or even rejected by the inmates. It is one reason why many inmates ask to be taken off the special diet.

There is therefore a major difference between the prescribed "special" diets and the number of diagnoses of metabolic diseases that require this type of diet, which shows that there is a problem of dietary under-prescription.
On the other hand, there are studies ${ }^{4,9}$ that affirm that increasing the variety of fresh fruit available at lunch, breakfast or afternoon snack can be a good option in preventing consumption of products from the prison shops, which takes place in over $95 \%$ of the surveyed inmates. In some Latin American countries, such as Colombia ${ }^{8}$, families are permitted to bring food into prison for the visits, and are generally typical meals; although in Spain the prison regulations prohibit this practice ${ }^{2}$, probably to ensure control and hygiene of foodstuffs and maintain equality of nutrition amongst the prison population. 
The conclusion is that the analysis by groups of foods of the meals offered by the CIRE shows that the diet needs changes, according to the IASE, since the score compared to the previous study in 2014 has hardly changed, despite recent changes in planning of planned menus and new cooking techniques mentioned in this article. It would therefore be advisable for the Dietetics and Nutrition Service of the CIRE to revise the menus, so as to correct the small deviations that were detected. These include reducing cured meats and pastries served on a daily basis, offering more variety in fruit and vegetables, increasing legumes as an option for the main course, and revising the thawing and cooking techniques of foods such as fish, eggs and meat to make them more palatable.

Another aspect requiring improvement is the food offered at the prison shops. It is suggested that products that are recommended for healthy eating should be included, such as sugar-free drinks, different types of fruit, fresh vegetables that can be eaten raw, brown bread and cereals, fresh cheese, etc.

As regards inmates' perceptions, the improvements made in the first quarter of 2017 have not been noted, either in terms of taste or quality of the meals.

Most of the medically prescribed diets drawn up before 2017 have been simplified by the CIRE in the form of one single diet, the "special diet", which covers all types of infectious and metabolic diseases. This means that any inmate who has one of these pathologies has to be fed with the same diet.

To sum up, the diet in prison needs to be improved. It is recommended that the food service of the CIRE and prison healthcare teams work together on this area. Implementing programmes to promote healthy habits, as suggested by other authors ${ }^{9}$, may also be beneficial.

\section{CORRESPONDENCE}

Ana Cristina Varoucha-Azcarate

Centro Penitenciario Quatre Camins.

La Roca del Vallès. Barcelona

E-mail: avaroucha@gmail.com

\section{REFERENCES}

1. Parrado MS. La alimentación en la cárcel provincial de Valladolid (1950-1951). Revista de Folklo- re. 1991;123. [fecha de acceso: 30 Ene 2017]. Disponible en: https://funjdiaz.net/folklore/O7ficha. php?id=961

2. Reglamento Penitenciario: Real Decreto 190/1996, de 9 de febrero, por el que se aprueba el Reglamento Penitenciario. BOE. 15 Feb 1996;40:5380435. pp. 56. [actualizado: 26 Mar 2011; fecha de acceso: 3 Feb 2017]. Disponible en: https://www. boe.es/buscar/act.php?id=BOE-A-1996-3307

3. Decret $329 / 2006$, de 5 de setembre, pel qual s'aprova el Reglamenté d'organització i funcionament dels serveis d'execució penal a Catalunya. 2006;4714: 37902-22. [fecha de acceso: 3 Feb 2017]. Disponible en: http://dogc.gencat.cat/ca/ pdogc_canals_interns/pdogc_resultats_fitxa/?doc umentId $=460252 \&$ language $=$ ca_ES\&action $=$ fitxa

4. Sáiz-Izquierdo ME, Fornons D, Medina FX, Aguilar-Martínez A. Análisis del servicio de comedor y la opinión de los usuarios en un centro penitenciario de Cataluña. Nutr Hosp. 2014;30:213-8.

5. Norte A I, Ortiz R. Calidad de la dieta española según el índice de alimentación saludable. Nutr Hosp. 2011;26:330-6.

6. Departament de Justícia. Serveis penitenciaris. 2017 Dades estadístiques. Descriptors estadistics. [fecha de acceso: 26 Abr 2017]. Disponible en: http://justicia.gencat.cat/ca/departament/Estadistiques/serveis_penitenciaris/

7. Fornons D. Comer en la prisión; la identidad alimentaria de los presos de la cárcel Modelo de Barcelona. Zainak. 2011;34:147-60. [fecha de acceso: 30 Ene 2017]. Disponible en: http://www.euskomedia.org/PDFAnlt/zainak/34/34147160.pdf.

8. Bejaranoo-Roncancio JJ, Celedón-Dangond CA, Socha-Gracia L. Alimentación penitenciaria: entre higiene y derechos. Rev Fac Med. 2015;63:527-35.

9. Gil-Delgado Y, Domínguez-Zamorano JA, Martínez-Sánchez-Suárez E. Valoración de los beneficios para la salud conseguidos mediante un programa nutricional dirigido a internos con factores de riesgo cardiovascular del Centro Penitenciario de Huelva. Rev Esp Sanid Penit. 2011;13:75-83. 